\title{
AARP
}

\section{Latinas and Beauty as We Age: A Cultural Reflection (2021) Annotated Questionnaire}

\begin{tabular}{|c|c|}
\hline Survey sponsor & AARP \\
\hline Survey/Data collection supplier & NORC at the University of Chicago \\
\hline Population represented & U.S. Latina women ages 18-plus \\
\hline Sample size & 1,$890 ; 1,018$ ages $18-49,872$ ages 50 -plus \\
\hline Mode of data collection & $\begin{array}{l}\text { AmeriSpeak online panel and telephone (RDD } \\
\text { landline and RDD cell phones); Dynata online panel }\end{array}$ \\
\hline $\begin{array}{l}\text { Type of sample (probability/non- } \\
\text { probability) }\end{array}$ & $\begin{array}{l}\text { Probability for AmeriSpeak; Non-probability for } \\
\text { Dynata }\end{array}$ \\
\hline Start and end dates of data collection & November 17, 2020 - December 15, 2020 \\
\hline $\begin{array}{l}\text { Margin of sampling error for total } \\
\text { Latina sample, including the design } \\
\text { effect }\end{array}$ & $+/-4.1$ percentage points at the $95 \%$ confidence level \\
\hline $\begin{array}{l}\text { Margin of sampling error for key } \\
\text { subgroups, including the design effect }\end{array}$ & $\begin{array}{l}+/-7.1 \text { percentage points at the } 95 \% \text { confidence level } \\
\text { for Latina women ages } 50 \text {-plus }\end{array}$ \\
\hline Weighting & $\begin{array}{l}\text { The data are weighted to the U.S. population of } \\
\text { women ages 18-plus by race/ethnicity by age, } \\
\text { race/ethnicity by region, race/ethnicity by education, } \\
\text { age by education, age by English proficiency, and } \\
\text { age by country of birth to benchmarks derived from } \\
\text { the March } 2020 \text { Current Population Survey, as well as } \\
\text { predicted values of survey variables from small area } \\
\text { estimation models. }\end{array}$ \\
\hline
\end{tabular}

NORC at the University of Chicago is an independent research institution that delivers reliable data and rigorous analysis to guide critical programmatic, business, and policy decisions. Since 1941, NORC has conducted groundbreaking studies, created and applied innovative methods and tools, and advanced principles of scientific integrity and collaboration. Today, government, corporate, and nonprofit clients around the world partner with NORC to transform increasingly complex information into useful knowledge.

Headquartered in downtown Chicago, NORC works in over 40 countries around the world, with additional offices on the University of Chicago campus, the DC metro area, Atlanta, Boston, Silicon Valley and San Francisco. Please visit www.norc.org for more information. 
Main

Q1. In general, how important was your beauty and personal grooming routine to you before the coronavirus pandemic?

[HALF SAMPLE ASKED RESPONSE OPTIONS IN REVERSE ORDER]

\begin{tabular}{|l|c|c|c|}
\hline NORC & & & \\
$11 / 17-12 / 15$ & All women & $18-49$ & $50+$ \\
\hline Very/Somewhat important & $\mathbf{8 6}$ & $\mathbf{8 4}$ & 90 \\
\hline Very important & 38 & 35 & 43 \\
\hline Somewhat important & $\mathbf{4 8}$ & $\mathbf{4 9}$ & 47 \\
\hline Not too/Not at all important & $\mathbf{1 4}$ & $\mathbf{1 6}$ & 10 \\
\hline Not too important & 12 & 13 & 9 \\
\hline Not at all important & 2 & 2 & 1 \\
\hline DON'T KNOW & - & - & - \\
\hline SKIPPED ON WEB/REFUSED & $*$ & $*$ & - \\
\hline N= & 1,890 & 1,018 & 872 \\
\hline
\end{tabular}

Q2. Since the coronavirus pandemic began, would you say your beauty and personal grooming routine has become more important to you, less important, or has there been no difference?

\begin{tabular}{|l|c|c|c|}
\hline NORC & All & & \\
$11 / 17-12 / 15$ & women & $18-49$ & $50+$ \\
\hline A lot/Somewhat more important & $\mathbf{2 4}$ & $\mathbf{2 7}$ & $\mathbf{1 9}$ \\
\hline A lot important & 12 & 14 & 9 \\
\hline Somewhat more important & 12 & 13 & 10 \\
\hline No difference & $\mathbf{4 0}$ & $\mathbf{3 4}$ & $\mathbf{4 9}$ \\
\hline Somewhat less important/A lot less important & $\mathbf{3 6}$ & $\mathbf{3 8}$ & $\mathbf{3 1}$ \\
\hline Somewhat less important & 27 & 29 & 24 \\
\hline A lot less important & 8 & 9 & 7 \\
\hline DON'T KNOW & - & - & - \\
\hline SKIPPED ON WEB/REFUSED & $*$ & 1 & $*$ \\
\hline N= & 1,890 & 1,018 & 872 \\
\hline
\end{tabular}


Q3. Since the coronavirus pandemic began, have you done each of the following more often, less often, or about as often as before?

\section{[GRID ITEMS RANDOMIZED]}

Focused on your health than your appearance

\begin{tabular}{|l|c|c|c|}
\hline NORC & & & \\
$11 / 17-12 / 15$ & All women & $18-49$ & $50+$ \\
\hline Much/Somewhat more often & $\mathbf{6 1}$ & $\mathbf{5 9}$ & 63 \\
\hline Much more often & $\mathbf{2 7}$ & 24 & 31 \\
\hline Somewhat more often & 34 & 34 & 33 \\
\hline No change & $\mathbf{3 0}$ & $\mathbf{2 9}$ & $\mathbf{3 3}$ \\
\hline Somewhat/Much less often & $\mathbf{8}$ & $\mathbf{1 1}$ & $\mathbf{3}$ \\
\hline Somewhat less often & 6 & 8 & 3 \\
\hline Much less often & 2 & 3 & 1 \\
\hline DON'T KNOW & - & - & - \\
\hline SKIPPED ON WEB/REFUSED & $*$ & 1 & $*$ \\
\hline N= & 1,890 & 1,018 & 872 \\
\hline
\end{tabular}

Cared about what people think about your appearance

\begin{tabular}{|l|c|c|c|}
\hline NORC & All women & $18-49$ & $50+$ \\
$11 / 17-12 / 15$ & $\mathbf{2 0}$ & $\mathbf{2 5}$ & $\mathbf{1 1}$ \\
\hline Much/Somewhat more often & 8 & 9 & 5 \\
\hline Much more often & 13 & 16 & 6 \\
\hline Somewhat more often & $\mathbf{4 3}$ & $\mathbf{4 0}$ & $\mathbf{5 0}$ \\
\hline No change & $\mathbf{3 6}$ & $\mathbf{3 5}$ & $\mathbf{3 8}$ \\
\hline Somewhat/Much less often & 19 & 18 & 21 \\
\hline Somewhat less often & 18 & 17 & 18 \\
\hline Much less often & - & - & - \\
\hline DON'T KNOW & $*$ & $*$ & $*$ \\
\hline SKIPPED ON WEB/REFUSED & 1,890 & 1,018 & 872 \\
\hline N= & & &
\end{tabular}

Been confident in your appearance

\begin{tabular}{|l|c|c|c|}
\hline NORC & All women & $18-49$ & $50+$ \\
\hline $11 / 17-12 / 15$ & $\mathbf{3 1}$ & $\mathbf{3 4}$ & $\mathbf{2 5}$ \\
\hline Much/Somewhat more often & 12 & 14 & 8 \\
\hline Much more often & 19 & 20 & 17 \\
\hline Somewhat more often & $\mathbf{4 3}$ & $\mathbf{3 6}$ & $\mathbf{5 6}$ \\
\hline No change & $\mathbf{2 6}$ & $\mathbf{2 9}$ & 19 \\
\hline Somewhat/Much less often & 20 & 22 & 18 \\
\hline Somewhat less often & 6 & 8 & 2 \\
\hline Much less often & - & - & - \\
\hline DON'T KNOW & $*$ & $*$ & $*$ \\
\hline SKIPPED ON WEB/REFUSED & 1,890 & 1,018 & 872 \\
\hline N= & & &
\end{tabular}


Focused on your inner peace than outer beauty

\begin{tabular}{|l|c|c|c|}
\hline NORC & & & \\
$11 / 17-12 / 15$ & All women & $18-49$ & $50+$ \\
\hline Much/Somewhat more often & $\mathbf{5 7}$ & $\mathbf{5 5}$ & $\mathbf{6 0}$ \\
\hline Much more often & $\mathbf{2 4}$ & 23 & 25 \\
\hline Somewhat more often & 33 & 32 & 35 \\
\hline No change & $\mathbf{3 3}$ & 32 & 35 \\
\hline Somewhat/Much less often & $\mathbf{1 0}$ & $\mathbf{1 3}$ & $\mathbf{4}$ \\
\hline Somewhat less often & 6 & 8 & 3 \\
\hline Much less often & 4 & 5 & 1 \\
\hline DON'T KNOW & - & - & - \\
\hline SKIPPED ON WEB/REFUSED & $*$ & 1 & $*$ \\
\hline N= & 1,890 & 1,018 & 872 \\
\hline
\end{tabular}

Felt pressure to appear youthful

\begin{tabular}{|l|c|c|c|}
\hline NORC & All women & $18-49$ & $50+$ \\
$11 / 17-12 / 15$ & $\mathbf{2 1}$ & $\mathbf{2 6}$ & $\mathbf{1 1}$ \\
\hline Much/Somewhat more often & 7 & 9 & 4 \\
\hline Much more often & 14 & 17 & 8 \\
\hline Somewhat more often & $\mathbf{5 3}$ & $\mathbf{4 9}$ & $\mathbf{5 9}$ \\
\hline No change & $\mathbf{2 6}$ & $\mathbf{2 4}$ & $\mathbf{2 9}$ \\
\hline Somewhat/Much less often & 14 & 12 & 17 \\
\hline Somewhat less often & 12 & 12 & 12 \\
\hline Much less often & - & - & - \\
\hline DON'T KNOW & $*$ & $*$ & $*$ \\
\hline SKIPPED ON WEB/REFUSED & 1,890 & 1,018 & 872 \\
\hline N= & & &
\end{tabular}

Felt obligated to follow conventional forms of beauty

\begin{tabular}{|l|c|c|c|}
\hline NORC & & & \\
$11 / 17-12 / 15$ & All women & $18-49$ & $50+$ \\
\hline Much/Somewhat more often & $\mathbf{1 8}$ & $\mathbf{2 1}$ & 10 \\
\hline Much more often & 5 & 6 & 3 \\
\hline Somewhat more often & 12 & 15 & 7 \\
\hline No change & $\mathbf{5 0}$ & $\mathbf{4 5}$ & $\mathbf{5 9}$ \\
\hline Somewhat/Much less often & $\mathbf{3 2}$ & $\mathbf{3 3}$ & $\mathbf{3 1}$ \\
\hline Somewhat less often & 19 & $\mathbf{2 0}$ & $\mathbf{1 7}$ \\
\hline Much less often & 14 & 14 & 14 \\
\hline DON'T KNOW & $*$ & - & $*$ \\
\hline SKIPPED ON WEB/REFUSED & $*$ & 1 & $*$ \\
\hline $\mathrm{N}=$ & 1,890 & 1,018 & 872 \\
\hline
\end{tabular}


Q4. Which of the following statements is closest to your opinion?

\begin{tabular}{|l|c|c|c|}
\hline $\begin{array}{l}\text { NORC } \\
11 / 17-12 / 15\end{array}$ & All women & $18-49$ & $50+$ \\
\hline $\begin{array}{l}\text { During the pandemic, you changed your personal } \\
\text { grooming of beauty routine but will go back to the way it } \\
\text { was when the outbreak is over }\end{array}$ & 24 & 27 & 19 \\
\hline $\begin{array}{l}\text { During the pandemic, you changed your personal } \\
\text { grooming or beauty routine and will keep at least some of } \\
\text { the changes even after the outbreak is over }\end{array}$ & 36 & 40 & 30 \\
\hline $\begin{array}{l}\text { You haven't changed your personal grooming or beauty } \\
\text { routine during the pandemic }\end{array}$ & 39 & 33 & 50 \\
\hline DON'T KNOW & $*$ & - & $*$ \\
\hline SKIPPED ON WEB/REFUSED & 1 & 1 & 1 \\
\hline N= & 1,890 & 1,018 & 872 \\
\hline
\end{tabular}

Q5. Since the start of the coronavirus pandemic, do you think the standards of beauty for women in the U.S. have been raised, lowered, or remained the same?

\begin{tabular}{|l|c|c|c|}
\hline NORC & & & \\
$11 / 17-12 / 15$ & All women & $18-49$ & $50+$ \\
\hline Raised a lot/somewhat & $\mathbf{1 8}$ & $\mathbf{2 3}$ & 10 \\
\hline Raised a lot & 5 & 7 & 3 \\
\hline Raised somewhat & 13 & 16 & 8 \\
\hline Remained the same & $\mathbf{4 5}$ & $\mathbf{4 5}$ & $\mathbf{4 6}$ \\
\hline Lowered somewhat/a lot & $\mathbf{3 6}$ & $\mathbf{3 2}$ & $\mathbf{4 3}$ \\
\hline Lowered somewhat & 30 & 27 & 36 \\
\hline Lowered a lot & 6 & 5 & 7 \\
\hline DON'T KNOW & $*$ & $*$ & $*$ \\
\hline SKIPPED ON WEB/REFUSED & $*$ & $*$ & $*$ \\
\hline $\mathrm{N}=$ & 1,890 & 1,018 & 872 \\
\hline
\end{tabular}




\section{AARP}

If raised a lot, raised somewhat, lowered somewhat, or lowered a lot in Q5

Q6. Which of the following statements is closer to your opinion?

[HALF SAMPLE ASKED RESPONSE OPTIONS IN REVERSE ORDER]

\begin{tabular}{|c|c|c|c|}
\hline $\begin{array}{l}\text { NORC } \\
11 / 17-12 / 15\end{array}$ & All women & $18-49$ & $50+$ \\
\hline $\begin{array}{l}\text { These new standards of beauty for women established } \\
\text { during the pandemic should go back to the way they were } \\
\text { before the pandemic began. }\end{array}$ & 44 & 38 & 55 \\
\hline $\begin{array}{l}\text { These new standards of beauty for women established } \\
\text { during the pandemic should continue after the pandemic } \\
\text { ends. }\end{array}$ & 54 & 60 & 44 \\
\hline DON'T KNOW & - & - & - \\
\hline SKIPPED ON WEB/REFUSED & 2 & 3 & 1 \\
\hline $\mathrm{N}=$ & 1,097 & 594 & 503 \\
\hline
\end{tabular}

Those who say standards of beauty for women have been raised a lot or raised somewhat in Q5

\begin{tabular}{|l|c|c|c|}
\hline $\begin{array}{l}\text { NORC } \\
11 / 17-12 / 15\end{array}$ & All women & $18-49$ & $50+$ \\
\hline $\begin{array}{l}\text { These new standards of beauty for women established } \\
\text { during the pandemic should go back to the way they were } \\
\text { before the pandemic began. }\end{array}$ & 45 & 46 & 44 \\
\hline $\begin{array}{l}\text { These new standards of beauty for women established } \\
\text { during the pandemic should continue after the pandemic } \\
\text { ends. }\end{array}$ & 53 & 53 & 55 \\
\hline DON'T KNOW & - & - & - \\
\hline SKIPPED ON WEB/REFUSED & 2 & 2 & 1 \\
\hline N= & 371 & 261 & 110 \\
\hline
\end{tabular}

Those who say standards of beauty for women have been lowered a lot or lowered somewhat in Q5

\begin{tabular}{|l|c|c|c|}
\hline $\begin{array}{l}\text { NORC } \\
11 / 17-12 / 15\end{array}$ & All women & $18-49$ & $50+$ \\
\hline $\begin{array}{l}\text { These new standards of beauty for women established } \\
\text { during the pandemic should go back to the way they were } \\
\text { before the pandemic began. }\end{array}$ & 43 & 32 & 57 \\
\hline $\begin{array}{l}\text { These new standards of beauty for women established } \\
\text { during the pandemic should continue after the pandemic } \\
\text { ends. }\end{array}$ & 55 & 65 & 41 \\
\hline DON'T KNOW & - & - & - \\
\hline SKIPPED ON WEB/REFUSED & 2 & 3 & 1 \\
\hline N= & 726 & 333 & 393 \\
\hline
\end{tabular}




\section{ANRP}

If these new standards of beauty for women established during the pandemic should go back to the way they were before the pandemic began in Q6

Q7. And how likely do you think it is that these new standards of beauty for women established during the pandemic will go back to the way they were before the pandemic began?

[HALF SAMPLE ASKED RESPONSE OPTIONS IN REVERSE ORDER]

\begin{tabular}{|l|c|c|c|}
\hline NORC & & & \\
$11 / 17-12 / 15$ & All women & $18-49$ & $50+$ \\
\hline Extremely/Very likely & $\mathbf{4 5}$ & $\mathbf{4 2}$ & $\mathbf{4 7}$ \\
\hline Extremely likely & 12 & 13 & 10 \\
\hline Very likely & 32 & 29 & 37 \\
\hline Somewhat likely & $\mathbf{4 3}$ & $\mathbf{4 2}$ & $\mathbf{4 4}$ \\
\hline Not very/Not at all likely & $\mathbf{1 2}$ & $\mathbf{1 6}$ & $\mathbf{8}$ \\
\hline Not very likely & 9 & 12 & 7 \\
\hline Not at all likely & 3 & 4 & 1 \\
\hline DON'T KNOW & - & - & - \\
\hline SKIPPED ON WEB/REFUSED & - & - & - \\
\hline N= & 432 & 226 & 206 \\
\hline
\end{tabular}

Those who say standards of beauty for women have been raised a lot or raised somewhat in Q5

\begin{tabular}{|l|c|c|c|}
\hline NORC & All women & $18-49$ & $50+^{*}$ \\
$11 / 17-12 / 15$ & $\mathbf{4 0}$ & $\mathbf{4 1}$ & \\
\hline Extremely/Very likely & 11 & 12 & \\
\hline Extremely likely & 29 & 29 & \\
\hline Very likely & $\mathbf{4 2}$ & $\mathbf{4 1}$ & \\
\hline Somewhat likely & $\mathbf{1 8}$ & $\mathbf{1 7}$ & \\
\hline Not very/Not at all likely & 13 & 11 & \\
\hline Not very likely & 5 & 6 & \\
\hline Not at all likely & - & - & \\
\hline DON'T KNOW & - & - & \\
\hline SKIPPED ON WEB/REFUSED & 167 & 127 & 40 \\
\hline N= & & \\
\hline
\end{tabular}

*Sample sizes below $n=100$, too small to analyze. 


\section{$\triangle A R P$}

Those who say standards of beauty for women have been lowered a lot or lowered somewhat in Q5

\begin{tabular}{|l|c|c|c|}
\hline NORC & & & \\
$11 / 17-12 / 15$ & All women & $18-49$ & $50+$ \\
\hline Extremely/Very likely & $\mathbf{4 7}$ & $\mathbf{4 3}$ & $\mathbf{5 0}$ \\
\hline Extremely likely & 13 & 15 & 11 \\
\hline Very likely & 34 & 29 & 39 \\
\hline Somewhat likely & $\mathbf{4 4}$ & $\mathbf{4 3}$ & $\mathbf{4 5}$ \\
\hline Not very/Not at all likely & $\mathbf{8}$ & $\mathbf{1 3}$ & $\mathbf{5}$ \\
\hline Not very likely & 7 & 12 & 4 \\
\hline Not at all likely & 2 & 2 & 1 \\
\hline DON'T KNOW & - & - & - \\
\hline SKIPPED ON WEB/REFUSED & - & - & - \\
\hline N= & 265 & 99 & 166 \\
\hline
\end{tabular}

If these new standards of beauty for women established during the pandemic should continue after the pandemic ends in Q6

Q8. And how likely do you think it is that these new standards of beauty for women established during the pandemic will continue after the pandemic ends?

[HALF SAMPLE ASKED RESPONSE OPTIONS IN REVERSE ORDER]

\begin{tabular}{|l|c|c|c|}
\hline NORC & & & \\
$11 / 17-12 / 15$ & All women & $18-49$ & $50+$ \\
\hline Extremely/Very likely & $\mathbf{3 1}$ & $\mathbf{3 2}$ & $\mathbf{2 9}$ \\
\hline Extremely likely & 11 & 12 & 7 \\
\hline Very likely & 20 & 20 & 21 \\
\hline Somewhat likely & $\mathbf{4 2}$ & $\mathbf{4 2}$ & $\mathbf{4 2}$ \\
\hline Not very/Not at all likely & $\mathbf{2 7}$ & $\mathbf{2 6}$ & $\mathbf{2 9}$ \\
\hline Not very likely & 21 & 19 & 26 \\
\hline Not at all likely & 6 & 7 & 3 \\
\hline DON'T KNOW & - & - & - \\
\hline SKIPPED ON WEB/REFUSED & $*$ & $*$ & $*$ \\
\hline N= & 650 & 356 & 294 \\
\hline
\end{tabular}


Those who say standards of beauty for women have been raised a lot or raised somewhat in Q5

\begin{tabular}{|l|c|c|c|}
\hline NORC & All women & $18-49$ & $50+^{*}$ \\
$11 / 17-12 / 15$ & $\mathbf{5 4}$ & $\mathbf{5 2}$ & \\
\hline Extremely/Very likely & 20 & 21 & \\
\hline Extremely likely & 33 & 31 & \\
\hline Very likely & $\mathbf{3 3}$ & $\mathbf{3 3}$ & \\
\hline Somewhat likely & $\mathbf{1 3}$ & $\mathbf{1 5}$ & \\
\hline Not very/Not at all likely & 12 & 13 & \\
\hline Not very likely & 2 & 2 & \\
\hline Not at all likely & - & - & \\
\hline DON'T KNOW & $*$ & - & \\
\hline SKIPPED ON WEB/REFUSED & 198 & 129 & 69 \\
\hline $\mathrm{N}=$ & &
\end{tabular}

*Sample sizes below $n=100$, too small to analyze.

Those who say standards of beauty for women have been lowered a lot or lowered somewhat in Q5

\begin{tabular}{|l|c|c|c|}
\hline NORC & & & \\
$11 / 17-12 / 15$ & All women & $18-49$ & $50+$ \\
\hline Extremely/Very likely & $\mathbf{2 0}$ & $\mathbf{2 1}$ & $\mathbf{1 8}$ \\
\hline Extremely likely & 6 & 7 & 4 \\
\hline Very likely & 14 & 14 & 14 \\
\hline Somewhat likely & $\mathbf{4 6}$ & $\mathbf{4 6}$ & $\mathbf{4 5}$ \\
\hline Not very/Not at all likely & $\mathbf{3 5}$ & $\mathbf{3 3}$ & $\mathbf{3 6}$ \\
\hline Not very likely & 26 & 22 & 32 \\
\hline Not at all likely & 8 & 10 & 4 \\
\hline DON'T KNOW & - & - & - \\
\hline SKIPPED ON WEB/REFUSED & $*$ & - & 1 \\
\hline N= & 452 & 227 & 225 \\
\hline
\end{tabular}

Q9. Since the coronavirus pandemic began, would you say you have become more self-conscious about your physical appearance, less self-conscious, or there is no difference?

\begin{tabular}{|l|c|c|c|}
\hline NORC & & & \\
$11 / 17-12 / 15$ & All women & $18-49$ & $50+$ \\
\hline A lot more/Somewhat more self-conscious & $\mathbf{2 9}$ & $\mathbf{3 3}$ & $\mathbf{2 1}$ \\
\hline A lot more self-conscious & 8 & 10 & 5 \\
\hline Somewhat more conscious & 21 & 24 & 16 \\
\hline No difference & $\mathbf{4 7}$ & $\mathbf{4 3}$ & $\mathbf{5 5}$ \\
\hline Somewhat/A lot less self-conscious & $\mathbf{2 3}$ & $\mathbf{2 2}$ & $\mathbf{2 4}$ \\
\hline Somewhat less self-conscious & 18 & 17 & 20 \\
\hline A lot less self-conscious & 5 & 5 & 3 \\
\hline DON'T KNOW & - & - & - \\
\hline SKIPPED ON WEB/REFUSED & 1 & 1 & $*$ \\
\hline N= & 1,890 & 1,018 & 872 \\
\hline
\end{tabular}


EMPLOY1. What statement best describes your current employment status?

\begin{tabular}{|l|c|c|c|}
\hline NORC & & & \\
$11 / 17-12 / 15$ & All women & $18-49$ & $50+$ \\
\hline Employed & 46 & 56 & 29 \\
\hline Working remotely as a paid employee & 12 & 14 & 8 \\
\hline Working in-person as a paid employee & 25 & 31 & 14 \\
\hline Working remotely - self-employed & 5 & 7 & 3 \\
\hline Working in-person - self-employed & 4 & 4 & 5 \\
\hline Not employed & 53 & 44 & 70 \\
\hline $\begin{array}{l}\text { Not working - laid off or furloughed since } \\
\text { the start of the coronavirus pandemic }\end{array}$ & 11 & & \\
\hline $\begin{array}{l}\text { Not working - unemployed looking for work } \\
\text { since before the coronavirus pandemic }\end{array}$ & 9 & 9 & 15 \\
\hline Not working - retired & 12 & $*$ & 83 \\
\hline Not working - disabled & 5 & 4 & 8 \\
\hline Not working - student & 4 & 5 & $*$ \\
\hline Not working - other & 13 & 16 & 7 \\
\hline DON'T KNOW & $*$ & - & $*$ \\
\hline SKIPPED ON WEB/REFUSED & $*$ & $*$ & $*$ \\
\hline N= & 1,890 & 1,018 & 872 \\
\hline
\end{tabular}

Q10. Have you felt pressure from any of the following to keep up with your physical appearance during the coronavirus pandemic?

\section{All women}

\begin{tabular}{|l|c|}
\hline NORC & Yes \\
\hline $11 / 17-12 / 15$ & 44 \\
\hline Yourself & 11 \\
\hline Spouse or partner & 14 \\
\hline Friends or other family & 2 \\
\hline $\begin{array}{l}\text { If working remotely or in-person as a paid employee } \\
\text { Bosses or managers }\end{array}$ & 3 \\
\hline $\begin{array}{l}\text { If working remotely or in-person as a paid employee } \\
\text { Co-workers }\end{array}$ & 15 \\
\hline Social media & 9 \\
\hline Media in general & 2 \\
\hline Church groups or other religious groups & 1 \\
\hline Other - please specify & 40 \\
\hline Have not felt pressure from any of these & - \\
\hline DON'T KNOW & $*$ \\
\hline SKIPPED ON WEB/REFUSED & \\
\hline N=1,890 & \\
\hline
\end{tabular}


$18-49$

\begin{tabular}{|l|c|}
\hline NORC & Yes \\
\hline $11 / 17-12 / 15$ & 49 \\
\hline Yourself & 14 \\
\hline Spouse or partner & 14 \\
\hline Friends or other family & 3 \\
\hline $\begin{array}{l}\text { If working remotely or in-person as a paid employee } \\
\text { Bosses or managers }\end{array}$ & \\
\hline $\begin{array}{l}\text { If working remotely or in-person as a paid employee } \\
\text { Co-workers }\end{array}$ & 4 \\
\hline Social media & 18 \\
\hline Media in general & 10 \\
\hline Church groups or other religious groups & 2 \\
\hline Other - please specify & 1 \\
\hline Have not felt pressure from any of these & 31 \\
\hline DON'T KNOW & $*$ \\
\hline SKIPPED ON WEB/REFUSED & \\
\hline N=1,018 & \\
\hline
\end{tabular}

50+

\begin{tabular}{|l|c|}
\hline NORC & Yes \\
11/17-12/15 & 33 \\
\hline Yourself & 6 \\
\hline Spouse or partner & 12 \\
\hline Friends or other family & $*$ \\
\hline $\begin{array}{l}\text { If working remotely or in-person as a paid employee } \\
\text { Bosses or managers }\end{array}$ & \\
\hline $\begin{array}{l}\text { If working remotely or in-person as a paid employee } \\
\text { Co-workers }\end{array}$ & 1 \\
\hline Social media & 8 \\
\hline Media in general & 1 \\
\hline Church groups or other religious groups & $*$ \\
\hline Other - please specify & 55 \\
\hline Have not felt pressure from any of these & - \\
\hline DON'T KNOW & \\
\hline SKIPPED ON WEB/REFUSED & \\
\hline N=872 & \\
\hline
\end{tabular}




\section{A ARP}

Q11. Since the start of the coronavirus pandemic, have you felt more pressure, less pressure, or experienced no change in pressure to do each of the following?

\section{[Randomize grid items]}

To post good photos of yourself on social media

\begin{tabular}{|l|c|c|c|}
\hline NORC & & & \\
$11 / 17-12 / 15$ & All women & $18-49$ & $50+$ \\
\hline A lot more/Somewhat pressure & $\mathbf{2 3}$ & $\mathbf{2 8}$ & $\mathbf{1 3}$ \\
\hline A lot more pressure & 8 & 11 & 2 \\
\hline Somewhat pressure & 15 & 17 & 11 \\
\hline No change in pressure & $\mathbf{5 9}$ & $\mathbf{5 5}$ & $\mathbf{6 6}$ \\
\hline Somewhat/A lot less pressure & $\mathbf{1 8}$ & $\mathbf{1 7}$ & $\mathbf{2 0}$ \\
\hline Somewhat less pressure & 8 & 10 & 6 \\
\hline A lot less pressure & 10 & 8 & 14 \\
\hline DON'T KNOW & $*$ & - & 1 \\
\hline SKIPPED ON WEB/REFUSED & $*$ & $*$ & 1 \\
\hline N= & 1,890 & 1,018 & 872 \\
\hline
\end{tabular}

To look a certain way

\begin{tabular}{|l|c|c|c|}
\hline NORC & & & \\
$11 / 17-12 / 15$ & All women & $18-49$ & $50+$ \\
\hline A lot more/Somewhat pressure & $\mathbf{2 3}$ & $\mathbf{2 8}$ & 14 \\
\hline A lot more pressure & 6 & 8 & 3 \\
\hline Somewhat pressure & 17 & 20 & 12 \\
\hline No change in pressure & $\mathbf{5 6}$ & 51 & 65 \\
\hline Somewhat/A lot less pressure & $\mathbf{2 1}$ & $\mathbf{2 1}$ & $\mathbf{2 1}$ \\
\hline Somewhat less pressure & 12 & 13 & 11 \\
\hline A lot less pressure & 9 & 8 & 10 \\
\hline DON’T KNOW & - & - & - \\
\hline SKIPPED ON WEB/REFUSED & $*$ & $*$ & $*$ \\
\hline N= & 1,890 & 1,018 & 872 \\
\hline
\end{tabular}

To wear clothing that is considered appropriate for your age

\begin{tabular}{|l|c|c|c|}
\hline NORC & & & \\
$11 / 17-12 / 15$ & All women & $18-49$ & $50+$ \\
\hline A lot more/Somewhat pressure & $\mathbf{1 9}$ & $\mathbf{2 3}$ & $\mathbf{1 1}$ \\
\hline A lot more pressure & 6 & 8 & 2 \\
\hline Somewhat pressure & 13 & 15 & 10 \\
\hline No change in pressure & $\mathbf{6 4}$ & $\mathbf{6 0}$ & $\mathbf{7 2}$ \\
\hline Somewhat/A lot less pressure & $\mathbf{1 7}$ & $\mathbf{1 7}$ & 16 \\
\hline Somewhat less pressure & $\mathbf{8}$ & 9 & 6 \\
\hline A lot less pressure & 9 & 8 & 10 \\
\hline DON'T KNOW & $*$ & - & $*$ \\
\hline SKIPPED ON WEB/REFUSED & $*$ & $*$ & $*$ \\
\hline N= & 1,890 & 1,018 & 872 \\
\hline
\end{tabular}




\section{ANRP}

To have hair that conforms to other people's standards

\begin{tabular}{|l|c|c|c|}
\hline NORC & & & \\
$11 / 17-12 / 15$ & All women & $18-49$ & $50+$ \\
\hline A lot more/Somewhat pressure & $\mathbf{2 2}$ & $\mathbf{2 6}$ & $\mathbf{1 4}$ \\
\hline A lot more pressure & 4 & 6 & 2 \\
\hline Somewhat pressure & 17 & 20 & 11 \\
\hline No change in pressure & $\mathbf{5 7}$ & $\mathbf{5 3}$ & $\mathbf{6 4}$ \\
\hline Somewhat/A lot less pressure & $\mathbf{2 1}$ & $\mathbf{2 1}$ & $\mathbf{2 2}$ \\
\hline Somewhat less pressure & 12 & 11 & 12 \\
\hline A lot less pressure & 10 & 10 & 10 \\
\hline DON'T KNOW & - & - & - \\
\hline SKIPPED ON WEB/REFUSED & $*$ & $*$ & $*$ \\
\hline N= & 1,890 & 1,018 & 872 \\
\hline
\end{tabular}

To wear makeup

\begin{tabular}{|l|c|c|c|}
\hline NORC & & & \\
$11 / 17-12 / 15$ & All women & $18-49$ & $50+$ \\
\hline A lot more/Somewhat pressure & $\mathbf{1 9}$ & $\mathbf{2 6}$ & $\mathbf{8}$ \\
\hline A lot more pressure & 5 & 7 & 2 \\
\hline Somewhat pressure & 14 & 19 & 6 \\
\hline No change in pressure & $\mathbf{5 1}$ & $\mathbf{4 5}$ & $\mathbf{6 3}$ \\
\hline Somewhat/A lot less pressure & $\mathbf{2 8}$ & $\mathbf{2 8}$ & $\mathbf{2 8}$ \\
\hline Somewhat less pressure & 16 & 17 & 13 \\
\hline A lot less pressure & 12 & 11 & 14 \\
\hline DON’T KNOW & $*$ & - & $*$ \\
\hline SKIPPED ON WEB/REFUSED & 1 & 1 & 2 \\
\hline N= & 1,890 & 1,018 & 872 \\
\hline
\end{tabular}

Q12. Since the coronavirus pandemic began, have you become more or less comfortable with each of the following, or has your comfort level not changed?

\section{[Randomize grid items]}

Letting your hair (or roots) go gray

\begin{tabular}{|l|c|c|c|}
\hline NORC & All women & $18-49$ & $50+$ \\
$11 / 17-12 / 15$ & 14 & 13 & 18 \\
\hline More comfortable now than before the pandemic & 50 & 44 & 60 \\
\hline No change since the pandemic began & 12 & 12 & 12 \\
\hline Less comfortable now that before the pandemic & 23 & 30 & 11 \\
\hline Not applicable & - & - & - \\
\hline DON'T KNOW & $*$ & $*$ & $*$ \\
\hline SKIPPED ON WEB/REFUSED & 1,890 & 1018 & 872 \\
\hline N $=$ & &
\end{tabular}


Wearing your hair natural

\begin{tabular}{|l|c|c|c|}
\hline NORC & & & \\
$11 / 17-12 / 15$ & All women & $18-49$ & $50+$ \\
\hline More comfortable now than before the pandemic & 24 & 26 & 19 \\
\hline No change since the pandemic began & 55 & 52 & 61 \\
\hline Less comfortable now that before the pandemic & 12 & 12 & 12 \\
\hline Not applicable & 9 & 9 & 8 \\
\hline DON'T KNOW & - & - & - \\
\hline SKIPPED ON WEB/REFUSED & $*$ & $*$ & $*$ \\
\hline N $=$ & 1,890 & 1,018 & 872 \\
\hline
\end{tabular}

Not having your nails done or manicured

\begin{tabular}{|l|c|c|c|}
\hline NORC & & & \\
$11 / 17-12 / 15$ & All women & $18-49$ & $50+$ \\
\hline More comfortable now than before the pandemic & 17 & 18 & 15 \\
\hline No change since the pandemic began & 49 & 48 & 50 \\
\hline Less comfortable now that before the pandemic & 17 & 19 & 14 \\
\hline Not applicable & 16 & 14 & 20 \\
\hline DON'T KNOW & - & - & - \\
\hline SKIPPED ON WEB/REFUSED & 1 & 1 & 1 \\
\hline N= & 1,890 & 1,018 & 872 \\
\hline
\end{tabular}

Q13. When doing errands outside your house since the coronavirus pandemic began, have you become more or less comfortable with each of the following, or has your comfort level not changed?

[Randomize grid items]

Wearing athletic clothing or loungewear

\begin{tabular}{|l|c|c|c|}
\hline NORC & & & \\
$11 / 17-12 / 15$ & All women & $18-49$ & $50+$ \\
\hline More comfortable now than before the pandemic & 36 & 39 & 31 \\
\hline No change since the pandemic began & 48 & 46 & 52 \\
\hline Less comfortable now that before the pandemic & 7 & 8 & 5 \\
\hline Not applicable & 8 & 6 & 11 \\
\hline DON'T KNOW & - & - & - \\
\hline SKIPPED ON WEB/REFUSED & 1 & 1 & 1 \\
\hline N= & 1,890 & 1,018 & 872 \\
\hline
\end{tabular}


Not having your hair done or styled

\begin{tabular}{|l|c|c|c|}
\hline NORC & & & \\
$11 / 17-12 / 15$ & All women & $18-49$ & $50+$ \\
\hline More comfortable now than before the pandemic & 24 & 27 & 19 \\
\hline No change since the pandemic began & 55 & 54 & 56 \\
\hline Less comfortable now that before the pandemic & 13 & 13 & 13 \\
\hline Not applicable & 8 & 6 & 12 \\
\hline DON'T KNOW & - & - & - \\
\hline SKIPPED ON WEB/REFUSED & 1 & 1 & 1 \\
\hline $\mathrm{N}=$ & 1,890 & 1,018 & 872 \\
\hline
\end{tabular}

Wearing head coverings like scarves, bandanas, turbans, or headwraps, bands, hats, or caps to cover your hair

\begin{tabular}{|l|c|c|c|}
\hline NORC & & & \\
$11 / 17-12 / 15$ & All women & $18-49$ & $50+$ \\
\hline More comfortable now than before the pandemic & 17 & 19 & 13 \\
\hline No change since the pandemic began & 47 & 45 & 50 \\
\hline Less comfortable now that before the pandemic & 9 & 8 & 9 \\
\hline Not applicable & 27 & 27 & 27 \\
\hline DON'T KNOW & - & - & - \\
\hline SKIPPED ON WEB/REFUSED & $*$ & $*$ & 1 \\
\hline $\mathrm{N}=$ & 1,890 & 1,018 & 872 \\
\hline
\end{tabular}

Not wearing make-up

\begin{tabular}{|l|c|c|c|}
\hline NORC & All women & $18-49$ & $50+$ \\
\hline $11 / 17-12 / 15$ & 29 & 33 & 22 \\
\hline More comfortable now than before the pandemic & 50 & 46 & 58 \\
\hline No change since the pandemic began & 12 & 12 & 10 \\
\hline Less comfortable now that before the pandemic & 9 & 8 & 10 \\
\hline Not applicable & - & - & - \\
\hline DON'T KNOW & 1 & 1 & $*$ \\
\hline SKIPPED ON WEB/REFUSED & 1,890 & 1,018 & 872 \\
\hline N= & &
\end{tabular}

Wearing clothing or make-up that expresses your culture

\begin{tabular}{|l|c|c|c|}
\hline NORC & & & \\
$11 / 17-12 / 15$ & All women & $18-49$ & $50+$ \\
\hline More comfortable now than before the pandemic & 11 & 14 & 7 \\
\hline No change since the pandemic began & 62 & 61 & 64 \\
\hline Less comfortable now that before the pandemic & 8 & 8 & 6 \\
\hline Not applicable & 19 & 17 & 22 \\
\hline DON'T KNOW & - & - & - \\
\hline SKIPPED ON WEB/REFUSED & 1 & 1 & $*$ \\
\hline N $=$ & 1,890 & 1,018 & 872 \\
\hline
\end{tabular}




\section{AARP}

Q14. When going to work or joining video calls with work colleagues since the coronavirus pandemic began, have you become more or less comfortable with each of the following, or has your comfort level not changed?

\section{[Randomize grid items]}

Wearing athletic clothing or loungewear

\begin{tabular}{|l|c|c|c|}
\hline NORC & & & \\
$11 / 17-12 / 15$ & All women & $18-49$ & $50+$ \\
\hline More comfortable now than before the pandemic & 34 & 35 & 27 \\
\hline No change since the pandemic began & 49 & 47 & 54 \\
\hline Less comfortable now that before the pandemic & 8 & 8 & 6 \\
\hline Not applicable & 9 & 8 & 12 \\
\hline DON'T KNOW & - & - & - \\
\hline SKIPPED ON WEB/REFUSED & $*$ & 1 & $*$ \\
\hline N $=$ & 964 & 630 & 334 \\
\hline
\end{tabular}

Not having your hair done or styled

\begin{tabular}{|l|c|c|c|}
\hline NORC & & & \\
$11 / 17-12 / 15$ & All women & $18-49$ & $50+$ \\
\hline More comfortable now than before the pandemic & 28 & 31 & 19 \\
\hline No change since the pandemic began & 51 & 50 & 56 \\
\hline Less comfortable now that before the pandemic & 11 & 11 & 13 \\
\hline Not applicable & 9 & 8 & 13 \\
\hline DON'T KNOW & - & - & - \\
\hline SKIPPED ON WEB/REFUSED & 1 & 1 & $*$ \\
\hline N $=$ & 964 & 630 & 334 \\
\hline
\end{tabular}

Wearing head coverings like scarves, bandanas, turbans, or headwraps, bands, hats, or caps to cover your hair

\begin{tabular}{|l|c|c|c|}
\hline NORC & All women & $18-49$ & $50+$ \\
$11 / 17-12 / 15$ & 16 & 16 & 14 \\
\hline More comfortable now than before the pandemic & 47 & 49 & 39 \\
\hline No change since the pandemic began & 7 & 5 & 14 \\
\hline Less comfortable now that before the pandemic & 29 & 29 & 32 \\
\hline Not applicable & - & - & - \\
\hline DON'T KNOW & $*$ & $*$ & $*$ \\
\hline SKIPPED ON WEB/REFUSED & 964 & 630 & 334 \\
\hline N $=$ & &
\end{tabular}


Not wearing make-up

\begin{tabular}{|l|c|c|c|}
\hline NORC & & & \\
$11 / 17-12 / 15$ & All women & $18-49$ & $50+$ \\
\hline More comfortable now than before the pandemic & 30 & 32 & 21 \\
\hline No change since the pandemic began & 49 & 49 & 49 \\
\hline Less comfortable now that before the pandemic & 12 & 11 & 17 \\
\hline Not applicable & 8 & 7 & 13 \\
\hline DON'T KNOW & - & - & - \\
\hline SKIPPED ON WEB/REFUSED & $*$ & 1 & $*$ \\
\hline N $=$ & 964 & 630 & 334 \\
\hline
\end{tabular}

Wearing clothing or make-up that expresses your culture

\begin{tabular}{|l|c|c|c|}
\hline NORC & & & \\
$11 / 17-12 / 15$ & All women & $18-49$ & $50+$ \\
\hline More comfortable now than before the pandemic & 18 & 20 & 12 \\
\hline No change since the pandemic began & 55 & 54 & 55 \\
\hline Less comfortable now that before the pandemic & 7 & 7 & 6 \\
\hline Not applicable & 20 & 19 & 26 \\
\hline DON'T KNOW & - & - & - \\
\hline SKIPPED ON WEB/REFUSED & $*$ & $*$ & $*$ \\
\hline N= & 964 & 630 & 334 \\
\hline
\end{tabular}

\section{Q15. Since the pandemic started, did you...?}

\begin{tabular}{|l|c|c|c|}
\hline NORC & & & \\
$11 / 17-12 / 15$ & All women & $18-49$ & $50+$ \\
\hline Continue to dye your hair & 39 & 32 & 51 \\
\hline Stop dyeing your hair & 18 & 19 & 17 \\
\hline Not dye your hair before the pandemic & 37 & 42 & 29 \\
\hline Start to dye your hair during the pandemic & 5 & 6 & 2 \\
\hline DON'T KNOW & - & - & - \\
\hline SKIPPED ON WEB/REFUSED & 1 & 1 & 1 \\
\hline N= & 1,890 & 1,018 & 872 \\
\hline
\end{tabular}




\section{AARP}

Q16. Since the coronavirus pandemic began, would you say you have used each of the following more often, less often, or neither more or less often?

[RANDOMIZE GRID ITEMS]

\section{All women}

\begin{tabular}{|l|c|c|c|c|c|}
\hline NORC & $\begin{array}{c}\text { More } \\
\text { often }\end{array}$ & $\begin{array}{c}\text { Less } \\
\text { often }\end{array}$ & $\begin{array}{c}\text { Neither more } \\
\text { or less often }\end{array}$ & $\begin{array}{c}\text { SKP/ } \\
\text { REF }\end{array}$ \\
\hline Facial skincare products, like serums or moisturizers & 30 & 21 & 49 & - & $*$ \\
\hline Body skincare products, like serums or moisturizers & 30 & 23 & 47 & - & 1 \\
\hline Hair products, like conditioners, oils, or hair-masks & 26 & 25 & 49 & - & $*$ \\
\hline Sunscreen or face or body & 15 & 34 & 50 & $*$ & 1 \\
\hline Eye makeup products & 14 & 43 & 43 & - & 1 \\
\hline Lipstick & 12 & 51 & 37 & $*$ & $*$ \\
\hline $\begin{array}{l}\text { Head scarves, bandanas, turbans, or headwraps, bands, } \\
\text { hats, caps to cover your hair }\end{array}$ & 21 & 24 & 55 & $*$ & $*$ \\
\hline $\begin{array}{l}\text { Styling tools like blow-dryers, straighteners, curling irons, } \\
\text { flexi or perm rods, or roller sets to style your hair }\end{array}$ & 13 & 40 & 47 & $*$ & 1 \\
\hline $\mathrm{N}=1,890$ & & & & & \\
\hline
\end{tabular}

\section{8-49}

\begin{tabular}{|l|c|c|c|c|c|}
\hline NORC & $\begin{array}{c}\text { More } \\
\text { often }\end{array}$ & $\begin{array}{c}\text { Less } \\
\text { often }\end{array}$ & $\begin{array}{c}\text { Neither more } \\
\text { or less often }\end{array}$ & DK & $\begin{array}{c}\text { SKP/ } \\
\text { REF }\end{array}$ \\
\hline Facial skincare products, like serums or moisturizers & 37 & 24 & 39 & - & $*$ \\
\hline Body skincare products, like serums or moisturizers & 35 & 27 & 38 & - & $*$ \\
\hline Hair products, like conditioners, oils, or hair-masks & 32 & 27 & 40 & - & $*$ \\
\hline Sunscreen or face or body & 18 & 38 & 43 & - & 1 \\
\hline Eye makeup products & 18 & 46 & 36 & - & 1 \\
\hline Lipstick & 16 & 50 & 33 & - & $*$ \\
\hline $\begin{array}{l}\text { Head scarves, bandanas, turbans, or headwraps, bands, } \\
\text { hats, caps to cover your hair }\end{array}$ & 24 & 22 & 54 & - & $*$ \\
\hline $\begin{array}{l}\text { Styling tools like blow-dryers, straighteners, curling irons, } \\
\text { flexi or perm rods, or roller sets to style your hair }\end{array}$ & 16 & 44 & 39 & - & \multirow{2}{*}{1} \\
\hline \begin{tabular}{l} 
N=1,018 \\
\hline
\end{tabular} & & & & \\
\hline
\end{tabular}


$50+$

\begin{tabular}{|l|c|c|c|c|c|}
\hline $\begin{array}{l}\text { NORC } \\
11 / 17-12 / 15\end{array}$ & $\begin{array}{c}\text { More } \\
\text { often }\end{array}$ & $\begin{array}{c}\text { Less } \\
\text { often }\end{array}$ & $\begin{array}{c}\text { Neither more } \\
\text { or less often }\end{array}$ & DK & $\begin{array}{c}\text { SKP/ } \\
\text { REF }\end{array}$ \\
\hline Facial skincare products, like serums or moisturizers & 16 & 17 & 67 & - & $*$ \\
\hline Body skincare products, like serums or moisturizers & 20 & 16 & 63 & - & 1 \\
\hline Hair products, like conditioners, oils, or hair-masks & 14 & 20 & 66 & - & $*$ \\
\hline Sunscreen or face or body & 9 & 28 & 63 & $*$ & $*$ \\
\hline Eye makeup products & 5 & 38 & 55 & - & 1 \\
\hline Lipstick & 4 & 51 & 45 & $*$ & $*$ \\
\hline $\begin{array}{l}\text { Head scarves, bandanas, turbans, or headwraps, bands, } \\
\text { hats, caps to cover your hair }\end{array}$ & 15 & 28 & 56 & $*$ & $*$ \\
\hline $\begin{array}{l}\text { Styling tools like blow-dryers, straighteners, curling irons, } \\
\text { flexi or perm rods, or roller sets to style your hair }\end{array}$ & 8 & 31 & 60 & $*$ & 1 \\
\hline $\mathrm{N}=872$ & & & & & \multirow{2}{*}{} \\
\hline
\end{tabular}

Q17. Since the coronavirus pandemic began, have you used a video conferencing application like Zoom, Skype, or FaceTime for any of the following?

\section{[RANDOMIZE GRID ITEMS]}

\section{All women}

\begin{tabular}{|l|l|}
\hline $\begin{array}{l}\text { NORC } \\
11 / 17-12 / 15\end{array}$ & Yes \\
\hline Keep in touch with friends or family & 55 \\
\hline $\begin{array}{l}\text { If employed in EMPLOY1 } \\
\text { Attend work meetings }\end{array}$ & 21 \\
\hline Attend church or other religious meetings & 17 \\
\hline $\begin{array}{l}\text { Attend other types of meetings such as support } \\
\text { groups, social groups, or community groups }\end{array}$ & 24 \\
\hline Haven't used a video conferencing application & 25 \\
\hline DON'T KNOW & - \\
\hline SKIPPED ON WEB/REFUSED & $*$ \\
\hline N=1,890 & \\
\hline
\end{tabular}

18-49

\begin{tabular}{|l|l|}
\hline $\begin{array}{l}\text { NORC } \\
11 / 17-12 / 15\end{array}$ & Yes \\
\hline Keep in touch with friends or family & 56 \\
\hline $\begin{array}{l}\text { If employed in EMPLOY1 } \\
\text { Attend work meetings }\end{array}$ & 25 \\
\hline Attend church or other religious meetings & 15 \\
\hline $\begin{array}{l}\text { Attend other types of meetings such as support } \\
\text { groups, social groups, or community groups }\end{array}$ & 28 \\
\hline Haven't used a video conferencing application & 19 \\
\hline DON'T KNOW & - \\
\hline SKIPPED ON WEB/REFUSED & $*$ \\
\hline N=1,018 & \\
\hline
\end{tabular}


$50+$

\begin{tabular}{|l|c|}
\hline NORC & Yes \\
\hline $11 / 17-12 / 15$ & 53 \\
\hline Keep in touch with friends or family & 13 \\
\hline $\begin{array}{l}\text { If employed in EMPLOY1 } \\
\text { Attend work meetings }\end{array}$ & 20 \\
\hline Attend church or other religious meetings & 17 \\
\hline $\begin{array}{l}\text { Attend other types of meetings such as support } \\
\text { groups, social groups, or community groups }\end{array}$ & 36 \\
\hline Haven't used a video conferencing application & - \\
\hline DON'T KNOW & $*$ \\
\hline SKIPPED ON WEB/REFUSED & - \\
\hline N=872
\end{tabular}

Q18. Compared to when you meet with people in-person, does using video conferencing applications make you more self-conscious about your appearance, less self-conscious, or is there no difference?

\begin{tabular}{|l|c|c|c|}
\hline NORC & & & \\
$11 / 17-12 / 15$ & All women & $18-49$ & $50+$ \\
\hline A lot more/Somewhat more self-conscious & 41 & 43 & 37 \\
\hline A lot more self-conscious & 14 & 15 & 11 \\
\hline Somewhat more conscious & 28 & 28 & 26 \\
\hline No difference & $\mathbf{4 4}$ & $\mathbf{4 1}$ & $\mathbf{5 1}$ \\
\hline Somewhat/A lot less self-conscious & $\mathbf{1 4}$ & $\mathbf{1 6}$ & $\mathbf{1 2}$ \\
\hline Somewhat less self-conscious & 11 & 12 & 10 \\
\hline A lot less self-conscious & 3 & 4 & 2 \\
\hline DON'T KNOW & - & - & - \\
\hline SKIPPED ON WEB/REFUSED & $*$ & $*$ & $*$ \\
\hline $\mathrm{N}=$ & 1,452 & 844 & 608 \\
\hline
\end{tabular}

Q20. Think about your own beauty and personal grooming routine. Have you experienced anything positive because of the pandemic?

\begin{tabular}{|l|c|c|c|}
\hline NORC & All women & $18-49$ & $50+$ \\
$11 / 17-12 / 15$ & 44 & 49 & 34 \\
\hline Yes & 56 & 50 & 66 \\
\hline No & - & - & - \\
\hline DON'T KNOW & $*$ & 1 & $*$ \\
\hline SKIPPED ON WEB/REFUSED & 1,890 & 1,018 & 872 \\
\hline $\mathrm{N}=$ & & & \\
\hline
\end{tabular}


If yes in Q20

Q20A. What is the most positive thing you have experienced?

Q21. And thinking about your own beauty and personal grooming routine, have you experienced anything negative because of the pandemic?

\begin{tabular}{|l|c|c|c|}
\hline NORC & All women & $18-49$ & $50+$ \\
$11 / 17-12 / 15$ & 33 & 36 & 27 \\
\hline Yes & 66 & 62 & 73 \\
\hline No & - & - & - \\
\hline DON'T KNOW & 1 & 1 & 1 \\
\hline SKIPPED ON WEB/REFUSED & 1,890 & 1,018 & 872 \\
\hline $\mathrm{N}=$ & \multicolumn{2}{|}{} \\
\hline
\end{tabular}

If yes in Q21

Q21A. What is the most negative thing you have experienced?

Q22. Think about the impact from the coronavirus pandemic on various aspects of your life, including the physical and mental health of you and your family, and the effect on your and your family's finances. Has worry or stress resulting from the pandemic caused you to experience any of the following, or not?

\section{[RANDOMIZE GRID ITEMS]}

All women

\begin{tabular}{|l|c|c|c|c|}
\hline NORC & Yes & No & DK & SKP/REF \\
\hline $11 / 17-12 / 15$ & 50 & 50 & - & $*$ \\
\hline Trouble falling asleep & 46 & 53 & - & 1 \\
\hline Trouble staying asleep & 32 & 68 & - & $*$ \\
\hline Sleeping too much & 24 & 76 & - & $*$ \\
\hline Poor appetite & 49 & 51 & - & $*$ \\
\hline Eating too much & 55 & 45 & - & $*$ \\
\hline Weight gain & 20 & 80 & - & 1 \\
\hline Weight loss & 30 & 69 & - & $*$ \\
\hline Hair loss or thinning & 30 & 70 & - & $*$ \\
\hline Teeth clenching, grinding or cracking & 38 & 61 & - & 1 \\
\hline Dry or itchy skin & 30 & 70 & - & $*$ \\
\hline Acne outbreak & & & & \\
\hline
\end{tabular}

$\mathrm{N}=1,890$ 


\begin{tabular}{|l|c|c|c|c|}
\hline NORC & Yes & No & DK & SKP/REF \\
11/17-12/15 & 53 & 47 & - & - \\
\hline Trouble falling asleep & 47 & 53 & - & 1 \\
\hline Trouble staying asleep & 35 & 65 & - & $*$ \\
\hline Sleeping too much & 27 & 73 & - & - \\
\hline Poor appetite & 51 & 49 & - & 1 \\
\hline Eating too much & 56 & 44 & - & $*$ \\
\hline Weight gain & 22 & 77 & - & 1 \\
\hline Weight loss & 32 & 68 & - & $*$ \\
\hline Hair loss or thinning & 32 & 68 & - & $*$ \\
\hline Teeth clenching, grinding or cracking & 38 & 60 & - & 1 \\
\hline Dry or itchy skin & 39 & 61 & - & $*$ \\
\hline Acne outbreak & & & & \\
\hline N=1,018 & & & & \\
\hline
\end{tabular}

50+

\begin{tabular}{|l|c|c|c|c|}
\hline NORC & Yes & No & DK & SKP/REF \\
\hline $11 / 17-12 / 15$ & 45 & 55 & - & $*$ \\
\hline Trouble falling asleep & 45 & 55 & - & $*$ \\
\hline Trouble staying asleep & 26 & 74 & - & $*$ \\
\hline Sleeping too much & 18 & 81 & - & 1 \\
\hline Poor appetite & 46 & 54 & - & $*$ \\
\hline Eating too much & 52 & 48 & - & $*$ \\
\hline Weight gain & 15 & 84 & - & 1 \\
\hline Weight loss & 29 & 72 & - & $*$ \\
\hline Hair loss or thinning & 27 & 74 & - & $*$ \\
\hline Teeth clenching, grinding or cracking & 38 & 62 & - & $*$ \\
\hline Dry or itchy skin & 13 & 87 & - & $*$ \\
\hline Acne outbreak & & & & \\
\hline N=872 & & & & \\
\hline
\end{tabular}




\section{AARP}

Q23. Many big, traditional beauty companies are now making and marketing products and services to include people with different hair types and skin tones. How much do you agree or disagree with the following statements?

\section{[RANDOMIZE GRID ITEMS]}

The expanded products and services for diverse people from big, traditional beauty companies are still too limited.

\begin{tabular}{|l|c|c|c|}
\hline NORC & All women & $18-49$ & $50+$ \\
$11 / 17-12 / 15$ & 39 & 46 & $\mathbf{2 7}$ \\
\hline Strongly/Somewhat agree & 12 & 14 & 7 \\
\hline Strongly agree & 27 & 32 & 19 \\
\hline Somewhat agree & 45 & 41 & 54 \\
\hline Neither agree nor disagree & 15 & 13 & 19 \\
\hline Somewhat/Strongly disagree & 12 & 10 & 16 \\
\hline Somewhat disagree & 3 & 3 & 3 \\
\hline Strongly disagree & $*$ & - & $*$ \\
\hline DON'T KNOW & $*$ & $*$ & 1 \\
\hline SKIPPED ON WEB/REFUSED & 1,890 & 1,018 & 872 \\
\hline N= & & &
\end{tabular}

The big, traditional beauty companies are only looking to make a profit.

\begin{tabular}{|l|c|c|c|}
\hline NORC & & & \\
$11 / 17-12 / 15$ & All women & $18-49$ & $50+$ \\
\hline Strongly/Somewhat agree & $\mathbf{6 0}$ & $\mathbf{5 9}$ & 60 \\
\hline Strongly agree & 23 & 24 & 23 \\
\hline Somewhat agree & 36 & 36 & 37 \\
\hline Neither agree nor disagree & $\mathbf{3 2}$ & $\mathbf{3 2}$ & $\mathbf{3 1}$ \\
\hline Somewhat/Strongly disagree & $\mathbf{8}$ & $\mathbf{8}$ & $\mathbf{8}$ \\
\hline Somewhat disagree & 6 & 7 & 6 \\
\hline Strongly disagree & 2 & 2 & 2 \\
\hline DON'T KNOW & $*$ & - & $*$ \\
\hline SKIPPED ON WEB/REFUSED & $*$ & $*$ & 1 \\
\hline N= & 1,890 & 1,018 & 872 \\
\hline
\end{tabular}




\section{ANRP}

The products and services for diverse people from big, traditional beauty companies are of inferior quality compared with their other products and services.

\begin{tabular}{|l|c|c|c|}
\hline NORC & All women & $18-49$ & $50+$ \\
$11 / 17-12 / 15$ & 29 & 37 & 16 \\
\hline Strongly/Somewhat agree & 9 & 10 & 6 \\
\hline Strongly agree & 21 & 27 & 10 \\
\hline Somewhat agree & 53 & 50 & 61 \\
\hline Neither agree nor disagree & 17 & 13 & 23 \\
\hline Somewhat/Strongly disagree & 11 & 9 & 15 \\
\hline Somewhat disagree & 6 & 4 & 8 \\
\hline Strongly disagree & $*$ & $*$ & $*$ \\
\hline DON'T KNOW & $*$ & $*$ & $*$ \\
\hline SKIPPED ON WEB/REFUSED & 1,890 & 1,018 & 872 \\
\hline N= & & &
\end{tabular}

Minority owned beauty brands provide better products and services for diverse people than traditional beauty brands like Maybelline or L'Oreal.

\begin{tabular}{|l|c|c|c|}
\hline NORC & & & \\
$11 / 17-12 / 15$ & All women & $18-49$ & $50+$ \\
\hline Strongly/Somewhat agree & 41 & 46 & 30 \\
\hline Strongly agree & 16 & 19 & 10 \\
\hline Somewhat agree & 25 & 27 & 20 \\
\hline Neither agree nor disagree & $\mathbf{4 7}$ & $\mathbf{4 4}$ & $\mathbf{5 2}$ \\
\hline Somewhat/Strongly disagree & $\mathbf{1 2}$ & $\mathbf{9}$ & $\mathbf{1 7}$ \\
\hline Somewhat disagree & 7 & 6 & 10 \\
\hline Strongly disagree & 5 & 4 & 7 \\
\hline DON'T KNOW & $*$ & $*$ & $*$ \\
\hline SKIPPED ON WEB/REFUSED & $*$ & $*$ & $*$ \\
\hline N= & 1,890 & 1,018 & 872 \\
\hline
\end{tabular}

You are more likely to buy from big, traditional beauty companies that are inclusive of diverse people.

\begin{tabular}{|l|c|c|c|}
\hline NORC & All women & $18-49$ & $50+$ \\
\hline $11 / 17-12 / 15$ & $\mathbf{4 1}$ & $\mathbf{4 5}$ & $\mathbf{3 5}$ \\
\hline Strongly/Somewhat agree & 14 & 16 & 10 \\
\hline Strongly agree & $\mathbf{2 7}$ & 28 & 25 \\
\hline Somewhat agree & $\mathbf{4 5}$ & $\mathbf{4 1}$ & $\mathbf{5 2}$ \\
\hline Neither agree nor disagree & $\mathbf{1 4}$ & $\mathbf{1 4}$ & $\mathbf{1 3}$ \\
\hline Somewhat/Strongly disagree & 9 & 10 & 8 \\
\hline Somewhat disagree & 5 & 4 & 5 \\
\hline Strongly disagree & $*$ & - & $*$ \\
\hline DON'T KNOW & $*$ & $*$ & $*$ \\
\hline SKIPPED ON WEB/REFUSED & 1,890 & 1,018 & 872 \\
\hline $\mathrm{N}=$ & & &
\end{tabular}




\section{AARP}

Q24. Do you rely on any home remedies to meet your beauty and personal grooming needs, or not?

\begin{tabular}{|l|c|c|c|}
\hline NORC & All women & $18-49$ & $50+$ \\
\hline $11 / 17-12 / 15$ & 40 & 42 & 36 \\
\hline Yes & 59 & 57 & 64 \\
\hline No & - & - & - \\
\hline DON'T KNOW & $*$ & 1 & $*$ \\
\hline SKIPPED ON WEB/REFUSED & 1,890 & 1,018 & 872 \\
\hline $\mathrm{N}=$ & & &
\end{tabular}

Q25. How much do you agree or disagree with the following statements about beauty and personal grooming products and services?

\section{[GRID ITEMS RANDOMIZED]}

There should be more beauty and personal grooming products and services tailored to people with your skin tone.

\begin{tabular}{|l|c|c|c|}
\hline NORC & All women & $18-49$ & $50+$ \\
\hline $11 / 17-12 / 15$ & 44 & 44 & 43 \\
\hline Strongly/Somewhat agree & 18 & 20 & 15 \\
\hline Strongly agree & 26 & 25 & 27 \\
\hline Somewhat agree & $\mathbf{4 5}$ & $\mathbf{4 4}$ & $\mathbf{4 5}$ \\
\hline Neither agree nor disagree & $\mathbf{1 1}$ & $\mathbf{1 1}$ & $\mathbf{1 1}$ \\
\hline Somewhat/Strongly disagree & 8 & 8 & 8 \\
\hline Somewhat disagree & 3 & 3 & 3 \\
\hline Strongly disagree & $*$ & - & $*$ \\
\hline DON'T KNOW & $*$ & $*$ & 1 \\
\hline SKIPPED ON WEB/REFUSED & 1,890 & 1,018 & 872 \\
\hline $\mathrm{N}=$
\end{tabular}

There should be more beauty and personal grooming products and services tailored to people with your hair type.

\begin{tabular}{|l|c|c|c|}
\hline NORC & All women & $18-49$ & $50+$ \\
$11 / 17-12 / 15$ & 46 & 47 & 44 \\
\hline Strongly/Somewhat agree & 21 & 22 & 19 \\
\hline Strongly agree & 25 & 25 & 24 \\
\hline Somewhat agree & $\mathbf{4 2}$ & $\mathbf{4 1}$ & $\mathbf{4 6}$ \\
\hline Neither agree nor disagree & $\mathbf{1 2}$ & $\mathbf{1 2}$ & 10 \\
\hline Somewhat/Strongly disagree & 8 & 9 & 7 \\
\hline Somewhat disagree & 3 & 3 & 4 \\
\hline Strongly disagree & $*$ & - & $*$ \\
\hline DON'T KNOW & $*$ & $*$ & $*$ \\
\hline SKIPPED ON WEB/REFUSED & 1,890 & 1,018 & 872 \\
\hline N= & & &
\end{tabular}




\section{AARP}

There should be more beauty and personal grooming products and services tailored to people your age.

\begin{tabular}{|c|c|c|c|}
\hline $\begin{array}{l}\text { NORC } \\
11 / 17-12 / 15\end{array}$ & All women & $18-49$ & $50+$ \\
\hline Strongly/Somewhat agree & 47 & 40 & 57 \\
\hline Strongly agree & 17 & 15 & 19 \\
\hline Somewhat agree & 30 & 25 & 39 \\
\hline Neither agree nor disagree & 41 & 46 & 33 \\
\hline Somewhat/Strongly disagree & 12 & 14 & 9 \\
\hline Somewhat disagree & 9 & 11 & 6 \\
\hline Strongly disagree & 3 & 3 & 3 \\
\hline DON'T KNOW & $*$ & - & $*$ \\
\hline SKIPPED ON WEB/REFUSED & * & $*$ & * \\
\hline $\mathrm{N}=$ & 1,890 & 1,018 & 872 \\
\hline
\end{tabular}

There should be more beauty and personal grooming products and services tailored to people to women going through perimenopause and/or menopause.

\begin{tabular}{|l|c|c|c|}
\hline NORC & All women & $18-49$ & $50+$ \\
\hline $11 / 17-12 / 15$ & $\mathbf{5 4}$ & $\mathbf{5 4}$ & $\mathbf{5 4}$ \\
\hline Strongly/Somewhat agree & 25 & 24 & 27 \\
\hline Strongly agree & 29 & 30 & 26 \\
\hline Somewhat agree & $\mathbf{4 0}$ & $\mathbf{4 0}$ & 39 \\
\hline Neither agree nor disagree & $\mathbf{6}$ & $\mathbf{6}$ & $\mathbf{8}$ \\
\hline Somewhat/Strongly disagree & 4 & 4 & 5 \\
\hline Somewhat disagree & 2 & 2 & 3 \\
\hline Strongly disagree & $*$ & - & $*$ \\
\hline DON'T KNOW & $*$ & $*$ & $*$ \\
\hline SKIPPED ON WEB/REFUSED & 1,890 & 1,018 & 872 \\
\hline $\mathrm{N}=$
\end{tabular}


Q26. What sources of information do you use to find out about beauty and personal grooming?

\section{All women}

\begin{tabular}{|l|c|}
\hline NORC & Yes \\
\hline $11 / 17-12 / 15$ & 32 \\
\hline Facebook & 34 \\
\hline Instagram & 8 \\
\hline Twitter & 22 \\
\hline Pinterest & 21 \\
\hline A search engine & 30 \\
\hline Television & 5 \\
\hline Newspaper & 6 \\
\hline Radio & 21 \\
\hline Magazines & 8 \\
\hline From a blog & 45 \\
\hline YouTube & 32 \\
\hline From friends or family (including in person, by phone, email, text or messaging apps) & 25 \\
\hline From people other than friends or family (e.g. hair dresser, nail technicians, etc.) & 8 \\
\hline Some other social media site & 11 \\
\hline None of the above & - \\
\hline DON'T KNOW & $*$ \\
\hline SKIPPED ON WEB/REFUSED & \\
\hline N=1,890 & \\
\hline
\end{tabular}

18-49

\begin{tabular}{|l|c|}
\hline NORC & Yes \\
\hline $11 / 17-12 / 15$ & 35 \\
\hline Facebook & 45 \\
\hline Instagram & 9 \\
\hline Twitter & 25 \\
\hline Pinterest & 22 \\
\hline A search engine & 25 \\
\hline Television & 3 \\
\hline Newspaper & 6 \\
\hline Radio & 16 \\
\hline Magazines & 9 \\
\hline From a blog & 53 \\
\hline YouTube & 28 \\
\hline From friends or family (including in person, by phone, email, text or messaging apps) & 21 \\
\hline From people other than friends or family (e.g. hair dresser, nail technicians, etc.) & 8 \\
\hline Some other social media site & 9 \\
\hline None of the above & - \\
\hline DON'T KNOW & $*$ \\
\hline SKIPPED ON WEB/REFUSED & \\
\hline N=1,018 & \\
\hline
\end{tabular}


$50+$

\begin{tabular}{|l|c|}
\hline NORC & Yes \\
\hline $11 / 17-12 / 15$ & 26 \\
\hline Facebook & 13 \\
\hline Instagram & 5 \\
\hline Twitter & 18 \\
\hline Pinterest & 19 \\
\hline A search engine & 40 \\
\hline Television & 9 \\
\hline Newspaper & 6 \\
\hline Radio & 30 \\
\hline Magazines & 6 \\
\hline From a blog & 28 \\
\hline YouTube & 38 \\
\hline From friends or family (including in person, by phone, email, text or messaging apps) & 31 \\
\hline From people other than friends or family (e.g. hair dresser, nail technicians, etc.) & 9 \\
\hline Some other social media site & 16 \\
\hline None of the above & - \\
\hline DON'T KNOW & $*$ \\
\hline SKIPPED ON WEB/REFUSED & - \\
\hline N=872 & \\
\hline
\end{tabular}

Q27. Since the coronavirus pandemic began, are you more likely to focus on media that emphasizes health over beauty, less likely, or has there been no change? By media, we mean print, online, social media, TV, etc.

\begin{tabular}{|l|c|c|c|}
\hline NORC & & & \\
$11 / 17-12 / 15$ & All women & $18-49$ & $50+$ \\
\hline A lot/Somewhat more likely & 38 & 39 & 35 \\
\hline A lot more likely & 10 & 11 & 10 \\
\hline Somewhat more likely & 27 & 28 & 25 \\
\hline No change & $\mathbf{5 3}$ & $\mathbf{5 1}$ & $\mathbf{5 6}$ \\
\hline Somewhat/A lot less likely & $\mathbf{9}$ & $\mathbf{9}$ & $\mathbf{9}$ \\
\hline Somewhat less likely & 5 & 5 & 5 \\
\hline A lot less likely & 4 & 4 & 4 \\
\hline DON'T KNOW & - & - & - \\
\hline SKIPPED ON WEB/REFUSED & $*$ & $*$ & $*$ \\
\hline $\mathrm{N}=$ & 1,890 & 1,018 & 872 \\
\hline
\end{tabular}


Q28. Since the coronavirus pandemic began how much money do you think you spend in a typical month on beauty/personal grooming products for yourself not for other people?

\begin{tabular}{|l|c|c|c|}
\hline NORC & All women & $18-49$ & $50+$ \\
$11 / 17-12 / 15$ & 16 & 16 & 16 \\
\hline$\$ 10$ or less & 20 & 17 & 25 \\
\hline \$1-\$25 & 26 & 25 & 27 \\
\hline$\$ 26-\$ 50$ & 18 & 20 & 15 \\
\hline$\$ 51-\$ 100$ & 14 & 15 & 11 \\
\hline More than \$100 & $\$ 30$ & $\$ 40$ & $\$ 30$ \\
\hline Median & 6 & 7 & 5 \\
\hline DON'T KNOW & $*$ & $*$ & $*$ \\
\hline SKIPPED ON WEB/REFUSED & 1,890 & 1,018 & 872 \\
\hline N= & & & \\
\hline
\end{tabular}

If entered a response other than don't know, skipped, or refused

Q29. Would you say this amount is more, less, or about the same as you spent in a typical month on beauty or personal grooming products for yourself before the pandemic began?

\begin{tabular}{|l|c|c|c|}
\hline NORC & & & \\
$11 / 17-12 / 15$ & All women & $18-49$ & $50+$ \\
\hline Spend a lot/a little more money now & $\mathbf{1 4}$ & $\mathbf{1 7}$ & $\mathbf{8}$ \\
\hline Spend a lot more money now & 4 & 4 & 3 \\
\hline Spend a little more money now & 10 & 13 & 5 \\
\hline Spend about the same amount now & $\mathbf{5 0}$ & $\mathbf{4 8}$ & $\mathbf{5 2}$ \\
\hline Spend a little/a lot less money now & $\mathbf{3 6}$ & $\mathbf{3 4}$ & $\mathbf{4 0}$ \\
\hline Spend a little less money now & 20 & 19 & 22 \\
\hline Spend a lot less money now & 16 & 15 & 18 \\
\hline DON'T KNOW & - & - & - \\
\hline SKIPPED ON WEB/REFUSED & $*$ & 1 & $*$ \\
\hline $\mathrm{N}=$ & 1,801 & 956 & 845 \\
\hline
\end{tabular}


Q30. On average about how many minutes do you spend on your beauty or personal grooming regimen, such as hair, skin, make-up, or shaving, in the morning? Please give your best estimate.

\begin{tabular}{|l|c|c|c|}
\hline NORC & & & \\
$11 / 17-12 / 15$ & All women & $18-49$ & $50+$ \\
\hline Less than 5 minutes & 15 & 15 & 14 \\
\hline $6-10$ minutes & 17 & 17 & 16 \\
\hline $11-15$ minutes & 12 & 10 & 14 \\
\hline $16-20$ minutes & 12 & 11 & 13 \\
\hline $21-25$ minutes & 9 & 8 & 9 \\
\hline $26-30$ minutes & 15 & 14 & 18 \\
\hline $31-35$ minutes & 1 & 1 & 1 \\
\hline More than 35 minutes & 15 & 14 & 13 \\
\hline Median & 20 & 20 & 20 \\
\hline DON'T KNOW & 5 & 7 & 2 \\
\hline SKIPPED ON WEB/REFUSED & - & - & - \\
\hline N= & 1,890 & 1,018 & 872 \\
\hline
\end{tabular}

Q31. On average about how many minutes do you spend on your beauty or personal grooming regimen, such as hair, skin, make-up, or shaving, in the evening? Please give your best estimate.

\begin{tabular}{|l|c|c|c|}
\hline NORC & & & \\
$11 / 17-12 / 15$ & All women & $18-49$ & $50+$ \\
\hline Less than 5 minutes & 17 & 17 & 16 \\
\hline $6-10$ minutes & 16 & 14 & 20 \\
\hline $11-15$ minutes & 13 & 13 & 13 \\
\hline $16-20$ minutes & 11 & 12 & 10 \\
\hline $21-25$ minutes & 13 & 12 & 14 \\
\hline $26-30$ minutes & 12 & 9 & 16 \\
\hline 31-35 minutes & 1 & 1 & 1 \\
\hline More than 35 minutes & 12 & 15 & 6 \\
\hline Median & 10 & 15 & 10 \\
\hline DON'T KNOW & 6 & 7 & 4 \\
\hline SKIPPED ON WEB/REFUSED & - & - & - \\
\hline N= & 1,890 & 1,018 & 872 \\
\hline
\end{tabular}




\section{AARP}

Q32. Overall, would you say the average amount of time you spend per day, that is morning and evening, on your beauty or personal grooming regimen is more, less, or the same as you spent in a typical day before the pandemic began?

\begin{tabular}{|l|c|c|c|}
\hline NORC & & & \\
$11 / 17-12 / 15$ & All women & $18-49$ & $50+$ \\
\hline Spend a lot/a little more time now & $\mathbf{2 0}$ & $\mathbf{2 5}$ & $\mathbf{1 1}$ \\
\hline Spend a lot more time now & $\mathbf{7}$ & 8 & 4 \\
\hline Spend a little more time now & 13 & 16 & 7 \\
\hline Spend about the same time now & $\mathbf{5 0}$ & $\mathbf{4 6}$ & $\mathbf{5 9}$ \\
\hline Spend a little/a lot less time now & $\mathbf{3 0}$ & $\mathbf{2 9}$ & $\mathbf{3 0}$ \\
\hline Spend a little less time now & 17 & 16 & 20 \\
\hline Spend a lot less time now & 12 & 13 & 11 \\
\hline DON'T KNOW & - & - & - \\
\hline SKIPPED ON WEB/REFUSED & $*$ & $*$ & - \\
\hline N= & 1,801 & 1,018 & 872 \\
\hline
\end{tabular}

Q33. How much do you agree or disagree with the following statement: I feel more comfortable in my own skin as I age.

\begin{tabular}{|l|c|c|c|}
\hline NORC & & & \\
$11 / 17-12 / 15$ & All women & $18-49$ & $50+$ \\
\hline Strongly/Somewhat agree & $\mathbf{6 9}$ & $\mathbf{6 4}$ & $\mathbf{7 8}$ \\
\hline Strongly agree & 26 & 23 & 31 \\
\hline Somewhat agree & 44 & 42 & 47 \\
\hline Somewhat/Strongly disagree & $\mathbf{3 0}$ & $\mathbf{3 5}$ & $\mathbf{2 1}$ \\
\hline Somewhat disagree & $\mathbf{2 4}$ & $\mathbf{2 7}$ & $\mathbf{1 7}$ \\
\hline Strongly disagree & 6 & 8 & 4 \\
\hline DON'T KNOW & - & - & - \\
\hline SKIPPED ON WEB/REFUSED & $*$ & 1 & $*$ \\
\hline N= & 1,890 & 1,018 & 872 \\
\hline
\end{tabular}


Q34. How much do you agree or disagree with the following statements about beauty and personal grooming ads?

\section{[RANDOMIZE GRID ITEMS]}

I wish beauty and personal grooming ads had more realistic images of women my age

\begin{tabular}{|l|c|c|c|}
\hline NORC & & & \\
$11 / 17-12 / 15$ & All women & $18-49$ & $50+$ \\
\hline Strongly/Somewhat agree & $\mathbf{8 6}$ & $\mathbf{8 2}$ & $\mathbf{9 2}$ \\
\hline Strongly agree & 44 & 42 & 47 \\
\hline Somewhat agree & 42 & 41 & 45 \\
\hline Somewhat/Strongly disagree & 14 & 17 & 8 \\
\hline Somewhat disagree & 12 & 15 & 7 \\
\hline Strongly disagree & 2 & 3 & 1 \\
\hline DON'T KNOW & $*$ & - & $*$ \\
\hline SKIPPED ON WEB/REFUSED & $*$ & $*$ & $*$ \\
\hline N= & 1,890 & 1,018 & 872 \\
\hline
\end{tabular}

Too often, the women in beauty and personal grooming ads are very young, even when the products are intended for older women.

\begin{tabular}{|l|c|c|c|}
\hline NORC & & & \\
$11 / 17-12 / 15$ & All women & $18-49$ & $50+$ \\
\hline Strongly/Somewhat agree & $\mathbf{8 4}$ & $\mathbf{8 0}$ & $\mathbf{9 2}$ \\
\hline Strongly agree & 43 & 39 & 51 \\
\hline Somewhat agree & 41 & 41 & 41 \\
\hline Somewhat/Strongly disagree & $\mathbf{1 6}$ & $\mathbf{2 0}$ & $\mathbf{8}$ \\
\hline Somewhat disagree & 13 & 16 & 7 \\
\hline Strongly disagree & 3 & 4 & 1 \\
\hline DON'T KNOW & - & - & - \\
\hline SKIPPED ON WEB/REFUSED & $*$ & $*$ & $*$ \\
\hline N= & 1,890 & 1,018 & 872 \\
\hline
\end{tabular}




\section{A ARP}

Q35. Now, think about ads in general - not just for beauty and personal grooming - how much do you agree or disagree with the following statements?

[RANDOMIZE GRID ITEMS]

Women my age are under-represented in ads

\begin{tabular}{|l|c|c|c|}
\hline NORC & & & \\
$11 / 17-12 / 15$ & All women & $18-49$ & $50+$ \\
\hline Strongly/Somewhat agree & $\mathbf{6 6}$ & $\mathbf{5 5}$ & $\mathbf{8 6}$ \\
\hline Strongly agree & 25 & 19 & 37 \\
\hline Somewhat agree & 41 & 37 & 48 \\
\hline Somewhat/Strongly disagree & $\mathbf{3 3}$ & $\mathbf{4 4}$ & 14 \\
\hline Somewhat disagree & 28 & 36 & 12 \\
\hline Strongly disagree & 5 & 7 & 2 \\
\hline DON'T KNOW & - & - & - \\
\hline SKIPPED ON WEB/REFUSED & 1 & 1 & $*$ \\
\hline N= & 1,890 & 1,018 & 872 \\
\hline
\end{tabular}

I prefer to buy from brands that feature a mix of ages in their ads

\begin{tabular}{|l|c|c|c|}
\hline NORC & & & \\
$11 / 17-12 / 15$ & All women & $18-49$ & $50+$ \\
\hline Strongly/Somewhat agree & $\mathbf{8 1}$ & $\mathbf{7 8}$ & $\mathbf{8 7}$ \\
\hline Strongly agree & 29 & 29 & 28 \\
\hline Somewhat agree & 52 & 49 & 58 \\
\hline Somewhat/Strongly disagree & $\mathbf{1 9}$ & $\mathbf{2 2}$ & 13 \\
\hline Somewhat disagree & 16 & 19 & 11 \\
\hline Strongly disagree & 2 & 2 & 2 \\
\hline DON'T KNOW & $*$ & - & $*$ \\
\hline SKIPPED ON WEB/REFUSED & 1 & 1 & $*$ \\
\hline N= & 1,890 & 1,018 & 872 \\
\hline
\end{tabular}

In ads, images of women my age reinforce outdated stereotypes

\begin{tabular}{|l|c|c|c|}
\hline NORC & & & \\
$11 / 17-12 / 15$ & All women & $18-49$ & $50+$ \\
\hline Strongly/Somewhat agree & $\mathbf{6 6}$ & $\mathbf{6 5}$ & $\mathbf{7 1}$ \\
\hline Strongly agree & 22 & 21 & 23 \\
\hline Somewhat agree & 44 & 43 & 47 \\
\hline Somewhat/Strongly disagree & $\mathbf{3 3}$ & $\mathbf{3 4}$ & $\mathbf{2 9}$ \\
\hline Somewhat disagree & 28 & 29 & 26 \\
\hline Strongly disagree & 4 & 5 & 4 \\
\hline DON'T KNOW & $*$ & - & 1 \\
\hline SKIPPED ON WEB/REFUSED & 1 & 1 & $*$ \\
\hline $\mathrm{N}=$ & 1,890 & 1,018 & 872 \\
\hline
\end{tabular}


C1. Where were you born?

\begin{tabular}{|l|c|c|c|}
\hline NORC & & & \\
$11 / 17-12 / 15$ & All women & $18-49$ & $50+$ \\
\hline In a U.S. state & 46 & 52 & 35 \\
\hline In Puerto Rico or another U.S. territory & 7 & 7 & 8 \\
\hline Outside the United States & 46 & 41 & 57 \\
\hline DON'T KNOW & - & - & - \\
\hline SKIPPED ON WEB/REFUSED & 1 & 1 & $*$ \\
\hline $\mathrm{N}=$ & 1,890 & 1,018 & 872 \\
\hline
\end{tabular}

If outside the United States in C1

C2. Where were you born?

\begin{tabular}{|l|c|c|c|}
\hline NORC & & & \\
$11 / 17-12 / 15$ & All women & $18-49$ & $50+$ \\
\hline Brazil & 2 & 2 & 2 \\
\hline Colombia & 7 & 7 & 7 \\
\hline Cuba & 14 & 6 & 26 \\
\hline Dominican Republic & 4 & 4 & 4 \\
\hline Ecuador & 1 & 2 & 1 \\
\hline El Salvador & 5 & 8 & 1 \\
\hline Germany & 1 & 1 & $*$ \\
\hline Ghana & 4 & 5 & 3 \\
\hline Jamaica & 1 & 1 & 1 \\
\hline Mexico & 39 & 46 & 30 \\
\hline Nigeria & $*$ & $*$ & - \\
\hline Peru & 2 & 2 & 2 \\
\hline Venezuela & 9 & 6 & 12 \\
\hline Other & 9 & 7 & 11 \\
\hline Don't know / NA & 2 & 3 & $*$ \\
\hline N= & 413 & 195 & 218 \\
\hline
\end{tabular}


Show if Hispanic

ENGLISHTALK. How well do you speak English?

\begin{tabular}{|l|c|c|c|}
\hline NORC & All women & $18-49$ & $50+$ \\
$11 / 17-12 / 15$ & $\mathbf{7 6}$ & $\mathbf{7 7}$ & $\mathbf{7 6}$ \\
\hline Very well/Well & 61 & 61 & 61 \\
\hline Very well & 16 & 16 & 15 \\
\hline Well & $\mathbf{2 3}$ & $\mathbf{2 2}$ & $\mathbf{2 3}$ \\
\hline Not well/Not at all & 19 & 20 & 17 \\
\hline Not well & 3 & 2 & 6 \\
\hline Not at all & - & - & - \\
\hline DON'T KNOW & 1 & 1 & 1 \\
\hline SKIPPED ON WEB/REFUSED & 1,890 & 1,018 & 872 \\
\hline $\mathrm{N}=$ & & & \\
\hline
\end{tabular}

Show if Hispanic

ENGLISHREAD. How well do you read English?

\begin{tabular}{|l|c|c|c|}
\hline NORC & All women & $18-49$ & $50+$ \\
\hline $11 / 17-12 / 15$ & $\mathbf{8 0}$ & $\mathbf{8 1}$ & $\mathbf{7 8}$ \\
\hline Very well/Well & 63 & 64 & 63 \\
\hline Very well & 17 & 17 & 16 \\
\hline Well & $\mathbf{1 8}$ & $\mathbf{1 8}$ & $\mathbf{1 9}$ \\
\hline Not well/Not at all & 17 & 16 & 18 \\
\hline Not well & 2 & 2 & 1 \\
\hline Not at all & - & - & - \\
\hline DON'T KNOW & 2 & 1 & 2 \\
\hline SKIPPED ON WEB/REFUSED & 1,890 & 1,018 & 872 \\
\hline $\mathrm{N}=$ & & & \\
\hline
\end{tabular}

LANG_OTHER1. Do you speak another language at home?

\begin{tabular}{|l|c|c|c|}
\hline NORC & All women & $18-49$ & $50+$ \\
$11 / 17-12 / 15$ & 75 & 73 & 78 \\
\hline Yes & 24 & 26 & 22 \\
\hline No & - & - & - \\
\hline DON'T KNOW & $*$ & 1 & $*$ \\
\hline SKIPPED ON WEB/REFUSED & 1,890 & 1,018 & 872 \\
\hline $\mathrm{N}=$ & \multicolumn{2}{|}{} \\
\hline
\end{tabular}


Show if yes in LANG_OTHER1

LANGOTHER2N. What is that language?

All women

\begin{tabular}{|l|c|}
\hline NORC & Yes \\
\hline $11 / 17-12 / 15$ & 93 \\
\hline Spanish & 1 \\
\hline Arabic & $*$ \\
\hline Chinese & 1 \\
\hline French & $*$ \\
\hline German & $*$ \\
\hline Korean & $*$ \\
\hline Polish & $*$ \\
\hline Russian & $*$ \\
\hline Tagalog & $*$ \\
\hline Vietnamese & 5 \\
\hline Other Language, Please specify & - \\
\hline DON’T KNOW & $*$ \\
\hline SKIPPED ON WEB/REFUSED & \\
\hline N=1,172 &
\end{tabular}

18-49

\begin{tabular}{|l|c|}
\hline NORC & Yes \\
\hline $11 / 17-12 / 15$ & 92 \\
\hline Spanish & 1 \\
\hline Arabic & 1 \\
\hline Chinese & 1 \\
\hline French & $*$ \\
\hline German & $*$ \\
\hline Korean & $*$ \\
\hline Polish & $*$ \\
\hline Russian & 1 \\
\hline Tagalog & - \\
\hline Vietnamese & 4 \\
\hline Other Language, Please specify & - \\
\hline DON’T KNOW & $*$ \\
\hline SKIPPED ON WEB/REFUSED & \\
\hline N=678 & \\
\hline
\end{tabular}


50+

\begin{tabular}{|l|c|}
\hline NORC & Yes \\
$11 / 17-12 / 15$ & 93 \\
\hline Spanish & - \\
\hline Arabic & - \\
\hline Chinese & 1 \\
\hline French & $*$ \\
\hline German & - \\
\hline Korean & $*$ \\
\hline Polish & - \\
\hline Russian & - \\
\hline Tagalog & $*$ \\
\hline Vietnamese & 5 \\
\hline Other Language, Please specify & - \\
\hline DON'T KNOW & - \\
\hline SKIPPED ON WEB/REFUSED & \\
\hline N=494 & \\
\hline
\end{tabular}

If employed in EMPLOY1

EMPLOY2A. During the coronavirus pandemic, has your job been considered:

\begin{tabular}{|l|c|c|c|}
\hline NORC & & & \\
$11 / 17-12 / 15$ & All women & $18-49$ & $50+$ \\
\hline Essential & 58 & 59 & 55 \\
\hline Non-essential & 29 & 27 & 36 \\
\hline Not sure & 13 & 14 & 9 \\
\hline SKIPPED ON WEB/REFUSED & - & - & - \\
\hline $\mathrm{N}=$ & 964 & 630 & 334 \\
\hline
\end{tabular}

LGBTQ. Which of the following best represents how you think of yourself?

\begin{tabular}{|l|c|c|c|}
\hline NORC & & & \\
$11 / 17-12 / 15$ & All women & $18-49$ & $50+$ \\
\hline Lesbian or gay & 2 & 3 & 1 \\
\hline Straight, that is, not lesbian or gay & 85 & 81 & 93 \\
\hline Bisexual & 8 & 11 & 2 \\
\hline SOMETHING ELSE & 5 & 5 & 3 \\
\hline DON'T KNOW & $*$ & - & $*$ \\
\hline SKIPPED ON WEB/REFUSED & $*$ & 1 & $*$ \\
\hline $\mathrm{N}=$ & 1,890 & 1,018 & 872 \\
\hline
\end{tabular}


AARP. Are you or your spouse a member of AARP?

\begin{tabular}{|l|c|c|c|}
\hline NORC & All women & $18-49$ & $50+$ \\
$11 / 17-12 / 15$ & 14 & 8 & 27 \\
\hline Yes & 85 & 92 & 73 \\
\hline No & $*$ & - & $*$ \\
\hline DON'T KNOW & $*$ & $*$ & $*$ \\
\hline SKIPPED ON WEB/REFUSED & 1,890 & 1,018 & 872 \\
\hline $\mathrm{N}=$ & \multicolumn{3}{|l}{} \\
\hline
\end{tabular}

AGE

\begin{tabular}{|l|c|c|c|}
\hline NORC & & & \\
$11 / 17-12 / 15$ & All women & $18-49$ & $50+$ \\
\hline $18-29$ & 28 & 43 & \\
\hline $30-39$ & 20 & 31 & \\
\hline $40-59$ & 35 & 26 & 52 \\
\hline $60-64$ & 7 & & 19 \\
\hline $65+$ & 10 & & 30 \\
\hline $\mathrm{N}=$ & 1,890 & 1,018 & 872 \\
\hline
\end{tabular}

\section{EDUCATION}

\begin{tabular}{|l|c|c|c|}
\hline NORC & All women & $18-49$ & $50+$ \\
$11 / 17-12 / 15$ & 19 & 17 & 21 \\
\hline Less than a high school diploma & 30 & 30 & 29 \\
\hline $\begin{array}{l}\text { High school graduate or } \\
\text { equivalent }\end{array}$ & 27 & 28 & 26 \\
\hline Some college & 24 & 24 & 23 \\
\hline College graduate or above & 1,890 & 1,018 & 872 \\
\hline $\mathrm{N}=$ & & & \\
\hline
\end{tabular}

\section{MARITAL STATUS}

\begin{tabular}{|l|c|c|c|}
\hline NORC & & & \\
$11 / 17-12 / 15$ & All women & $18-49$ & $50+$ \\
\hline Married & 41 & 38 & 47 \\
\hline Widowed & 5 & 2 & 11 \\
\hline Divorced & 8 & 4 & 15 \\
\hline Separated & 8 & 5 & 13 \\
\hline Never married & 25 & 34 & 9 \\
\hline Living with partner & 13 & 17 & 5 \\
\hline $\mathrm{N}=$ & 1,890 & 1,018 & 872 \\
\hline
\end{tabular}


EMPLOYMENT STATUS

\begin{tabular}{|l|c|c|c|}
\hline NORC & & & \\
$11 / 17-12 / 15$ & All women & $18-49$ & $50+$ \\
\hline Employed & 46 & 56 & 29 \\
\hline Not employed & 54 & 44 & 70 \\
\hline $\mathrm{N}=$ & 1,890 & 1,018 & 872 \\
\hline
\end{tabular}

\section{INCOME}

\begin{tabular}{|l|c|c|c|}
\hline NORC & & & \\
$11 / 17-12 / 15$ & All women & $18-49$ & $50+$ \\
\hline Under $\$ 10,000$ & 11 & 13 & 8 \\
\hline$\$ 10,000$ to under $\$ 20,000$ & 14 & 14 & 14 \\
\hline$\$ 20,000$ to under $\$ 30,000$ & 20 & 20 & 19 \\
\hline$\$ 30,000$ to under $\$ 40,000$ & 13 & 13 & 12 \\
\hline$\$ 40,000$ to under $\$ 50,000$ & 9 & 9 & 10 \\
\hline$\$ 50,000$ to under $\$ 75,000$ & 17 & 16 & 19 \\
\hline$\$ 75,000$ to under $\$ 100,000$ & 7 & 7 & 7 \\
\hline$\$ 100,000$ to under $\$ 150,000$ & 6 & 6 & 8 \\
\hline$\$ 150,000$ or more & 3 & 3 & 3 \\
\hline $\mathrm{N}=$ & 1,890 & 1,018 & 872 \\
\hline
\end{tabular}

